\title{
Socio-digital-visualisations as Positive- reinforcement Mechanisms for Encouraging Pro-social and Constructive Behaviours
}

\author{
Dion Rezki \\ Learning Technology Research Institute (LTRI) \\ London Metropolitan University \\ diondionart@gmail.com
}

\section{INTRODUCTION}

In this digital and information age we find ourselves in the "networked" self is quickly becoming an aggregator of 'information flows'(Castells 2005). It's becoming increasingly easier to quantify this networked self with the growing implementation of, mobile, web and smart-technologies. I would like to explore the relationship between complex networks and socio-digital-visualisation tools as a potential mechanism in assisting individual agents and networks with pro-social, constructive decision making.

The accelerating development and visualisedcomplexity of the globalised world is an area ripe with new possibilities for understanding the emergence of new levels of human behaviour, and the self-organisation of society .Data visualisation is in itself, an emergent field (Gaffney \& Young 2014), which makes the often imperceptible complexity of the world and it's networked structures, more easily assimilated and utilized for the purpose of social and global development.

"Modern sensing technology and cheap displays are increasingly being deployed in public social spaces. Fluid social spaces, where individuals come together, small groups form and re-form for the purpose of social interaction, are important to our communities. Whether these technologies will facilitate or hinder our social engagement is a function of the interaction design, the settings in which they are deployed, and the content on display. These three key aspects - Control, Context, and Content - are critical to how these technologies impact our social spaces and whether they are ultimately accepted or rejected." (McDonald et al. 2008)

\section{COMPLEXITY}

The underlying structure and integral nature of complexity from a sociological and network-science perspective, can be summarized with the phrase

"The whole is often greater than the sum of the parts."

Network-thinking and network-visualisation helps provide a new visual language for expressing both the commonalities and factors which influence other disparate areas found across the different types of Interdependent networks that collectively form the structure of both the natural and manmade world (Mitchell 2009). E.g. Biological, social, economic, ecological, information and infrastructure to name a few kinds of interdependent networks.

Encouraging a comprehensive understanding of how the myriad of individual human actions sequentially influences the overall dynamics and behaviour of the various networks that formulate the world can potentially assist in providing an aerial view of the interconnectivity and implications of all human action. When applied with the purposes of social and global development, the visualised implications of human actions can be more efficiently realized and utilized to encourage pro-social behaviours. Pro-social behaviours can be characterized as those intended to benefit not only other people and groups of people, but also behaviours that benefit the structural integrity and the quality of the various environments people inhabit (Brief \& Motowidlo 1986).

An area I would like to see explored and developed with more depth would be the potential applications in which complex- networks can be visualised and displayed, with optimal user-interactivity and within various public and private spaces to assist in 
developing our spatial and perceptual awareness of the interconnectivity of human actions and information flows across multiple networks and scales. Combing insights from multidisciplinary fields and framed within digital-ambient-intelligence applications, could potentially assist with encouraging pro-social behaviours and in turn, shaping the emergence of new levels of self \& social organisation.

\section{REFERENCES}

Castells, M. (2005) Space of flows, space of places: Materials for a theory of urbanism in the information age. Comparative planning cultures, 45-63.

Gaffney, O., and Young, D. (2014) Data visualisation for science: the next frontier? Future
Earth Home, http://www.futureearth.info/blog/2014jan-27/data-visualisation-science-next-frontier (retrieved 21 March 2014).

McDonald, D. W., McCarthy, J. F., Soroczak, S., Nguyen, D. H., and Rashid, A. M. (2008) Proactive displays: Supporting awareness in fluid social environments. I. ACM Transactions on ComputerHuman Interaction (TOCHI), 14(4), 16.

Mitchell, M. (2009) Complexity: A guided tour. Oxford University Press.

Brief, A. P., and Motowidlo, S. J. (1986) Prosocial organizational behaviors. Academy of management review, 11(4), 710-725. 